\title{
Efficacy of Intracavernosal Injections of 50-Unit versus 100-Unit Doses of AbobotulinumtoxinA (Masport@) in Vasculogenic Erectile Dysfunction with Phosphodiesterase Type 5 Inhibitors Resistant
}

\author{
Sajad Moradi ${ }^{1}$, Dinyar Khazaeli ${ }^{1}$, Mohammadreza Dadfar ${ }^{1}$ and Nima Bakhtiari (iD) ${ }^{2,}$ \\ ${ }^{1}$ Department of Urology, Faculty of Medicine, Ahvaz Jundishapur University of Medical Sciences, Ahvaz, Iran \\ ${ }^{2}$ Pain Research Center, Imam Khomeini Hospital, Ahvaz Jundishapur University of Medical Sciences, Ahvaz, Iran \\ "Corresponding author: Pain Research Center, Imam Khomeini Hospital, Ahvaz Jondishapur University of Medical Sciences, Ahvaz, Iran. Email: bakhtiari.n@ajums.ac.ir
}

Received 2021 September 10; Revised 2021 November 07; Accepted 2021 November 07.

\begin{abstract}
Background: We aimed to evaluate the safety and efficacy of 50-unit dose against 100-unit dose of intracavernosal injection (ICI) of AbobotulinumtoxinA (BTX-A) (Masport@ ) in patients with vascular erectile dysfunction (ED) resistant to first-line therapies, including phosphodiesterase type 5 inhibitors (PDE5I).

Methods: In this double-blind randomized controlled trial(RCT), 40 patients with ED resistant to PDE5I were randomly divided into two groups: ICI of a single dose of Masport @ 50 units and single dose of 100 units. Peak systolic velocity (PSV) confirmed arterial insufficiency vascular disorder. For all patients, IIEF (International Index of Erectile Function), SHIM (Sexual Health Inventory for Men), and EHS (Erection Hardness Score) questionnaires were completed. Six weeks after the treatment, the subjects were re-examined. Results: Our results showed an acceptable clinical efficacy and safety of ICI of Masport@ six weeks after injection. No systemic complications in patients were seen. Three patients complained of brief penile pain shortly after injection, but there were no other local complications. The increase in mean PSV in the 100-unit group due to treatment was significant (P-value $<0.0001$ ). Also, there was a significant difference between the two groups of 50- and 100-unit (P-value $<0.0001)$. In addition, the increase in mean IIEF-EF, SHIM score, and EHS due to treatment was significant between the two groups. For the 100-unit group, P-value $<0.0001$ and the difference between the two groups was also significant (P-value $<0.0001$ ), which indicated a better response to treatment in the 100-unit group. The mean increase of IIEF score (EF domain) was 4.3 (mean IIEF: 9.4 and 13.7 after and before, respectively) in the 100-unit group and (mean IIEF: 8.1 and 9.1 after and before, respectively) in the 50-unit group.

Conclusions: The results of this study showed that ICI of AbobotulinumtoxinA, especially at a dose of 100 units, in patients with refractory vasculogenic ED is safe and effective in improving sexual function and ultrasound indices.
\end{abstract}

Keywords: AbobotulinumtoxinA, Intracavernosal Injections, Erectile Dysfunction

\section{Background}

Erectile dysfunction (ED) is defined as the inability to obtain or maintain an adequate erection for sexual intercourse and is a complication that increases with age (1). The Massachusetts Male Aging Study (MMAS) reports an ED prevalence of about 50\%, 60\%, and 70\% in men aged 50 , 60 , and 70 years, respectively (2). There are several factors involved in the sexual function of older men, including physical and mental conditions, comorbidities, and medications used to treat them (3). The pathophysiology of ED may include vascular, neurological, psychological, or drug-induced factors (4). There is convincing evidence that the most important underlying cause is vascular ED, and that cardiovascular disease and ED have a common etiol- ogy and pathophysiology (5).

Risk factors for ED include age, smoking, diabetes mellitus (DM), hypertension, dyslipidemia, metabolic diseases, obesity, and sedentary lifestyle (1). The first line of ED treatment includes psychosexual counseling and oral therapy with phosphodiesterase 5 inhibitors (PDE5I), including sildenafil, vardenafil, tadalafil, and evanafil (6).

Unfortunately, effective treatment for complex ED is still evolving despite the convenience of oral therapy. Some patients have contraindications regarding the use of PDE5I, such as concomitant use of nitrate. Some patients cannot tolerate headaches, visual and auditory changes. Also, $11-44 \%$ of patients do not respond to monotherapy with PDE5I. For this group of patients, the second line of treatment includes vacuum and ICI of vasoactive 
prostanoids. Other methods, including adrenoceptor antagonists, central dopamine agonists, androgen replacement therapies, and other experimental drugs, have been used by physicians before surgery. However, satisfactory treatments for ED are still unknown and challenging (7).

The use of botulinum toxin in urology is not a new issue, but it is time for its widespread and proven use. Although it was first safely used on humans in 1970, the need for botulinum toxin in urology was not recognized until the first decade of the twentieth century. The use of type A botulinum toxin was transformed and developed into a wide range of non-invasive treatment modalities in the treatment of a wide range of urinary and sexual disorders. The mechanism of action of botulinum toxin is similar in different urological applications and is due to the presynaptic binding to the terminals of cholinergic nerves which reduces acetylcholine leading to neuromuscular block and relaxes the muscles at the injection site (8). In recent studies, researchers have used this muscle relaxant property of injecting botulinum toxin into the corpus cavernosa to increase penile erection as a new line of therapy in ED $(9,10)$. There are seven different serotypes of botulinum toxin. Commercially available types include serotypes A and B for human use, but their urological use is limited. Types used in urological studies include type A, brand BOTOX® in the United States, and brand Dysport $®$ Known in France (9).

In the urological use of the toxin, several studies have shown that its use is safe and systemic spread of the toxin does not appear to exist (6).

Because the pathophysiology and the reason for patients' lack of response to PDE5I is insufficient smooth muscle relaxation and veno-occlusive disorder (VOD), any treatment that increases cavernosal smooth muscle relaxation can increase the patient's response to PDE5I (10).

\section{Objectives}

Due to the lack of human and animal studies on the possible role of botulinum toxin in patients with refractory ED, this study was carried out to evaluate the safety and efficacy of 50-unit dose against 100-unit dose of intracavernosal injection (ICI) of AbobotulinumtoxinA (BTX-A) (Masport@) in patients with vascular ED resistant to firstline therapies, including PDE5I.

\section{Methods}

\subsection{Case selection}

In this double-blind, randomized controlled trial (RCT), we enrolled 40 patients referred to Imam Khomeini
Hospital of Ahvaz, Iran, from May 2019 to July 2020. All patients had ED and were resistant to oral PDE5I. Color Doppler examination of cavernosal vessels with $40 \mathrm{mg}$ papaverine injection was performed, and peak systolic velocity (PSV) was measured; the results confirmed arterial insufficiency vascular disorder. An informed consent was obtained from all participants prior to their inclusion in the study.

The inclusion criteria were: men aged 30 - 70 years old; having vascular ED proved by penile color Doppler ultrasound; and failure in response to first-line treatments. Meanwhile, the exclusion criteria were: significant cardiovascular disease that interferes with sexual activity; history of unstable physical and mental states; anatomical abnormality of the penis (eg, penile fibrosis) that impairs erectile function; hormonal disorders; and history of spinal or pelvic radical surgery.

\subsection{Questionnaires and Groups}

For all patients, IIEF (International Index of Erectile Function), SHIM (Sexual Health Inventory for Men), and EHS (Erection Hardness Score) questionnaires were completed. The patients were randomly divided into two equal groups ( $n=20$ in each group) as follows: ICI of a single-dose of Masport@ 100 units and single-dose of 50 units. In order to blind the study, only the injector was aware of the injected dose. Hence, the sonographer, the data collector, and the patients were not aware of the injection.

\subsection{Procedure and Safety}

One week after color Doppler ultrasound with papaverine injection, the patients lied down in supine position. After cleansing the skin with alcohol, intracavernosal injection was performed and the injection site was pressed directly for two minutes. To prevent systemic toxicity, a ring was used at the base of the penis at the time of injection and was kept in place for 15 minutes after injection. Patients were also subjected to cardiopulmonary monitoring up to 30 minutes after injection.

\subsection{Variables}

Patients were then advised to continue their PDE5I after intracavernosal treatment; in fact, Masport was an addon treatment. Six weeks after the treatment, the subjects were re-examined and penile color Doppler ultrasound with papaverine injection was done, and mean PSV was measured. Then, IIEF, SHIM, and EHS questionnaires were completed, and the response to treatment was evaluated and compared. 


\subsection{Ethical Considerations}

This study was approved by the Ethical Committee of Ahvaz Jundishapur University of Medical Sciences, Iran (code: IR.AJUMS.REC.1399.105). This study was performed on patients with refractory ED. A checklist with no names was used to collect information. Also, an informed consent was obtained from all patients. The participants were informed that their personal information is completely confidential and only the results are presented in general. The systemic and local effects of intracavernosal Masport injection were fully explained to the patients. The study received no funding.

\section{Results}

\subsection{Demographic Information}

The mean age of patients was 48.9 and 50.4 years in the 100-unit and 50-unit groups, respectively. There was no significant difference between the two groups in terms of mean age $(P$-value $=0.637)$. The mean duration of ED in patients was 3.6 years. Patients were categorized according to the severity of ED under treatment at baseline prior to abobotulinumtoxinA IC injections based on their EF-IIEF domain score (11). All patients in this study had severe vasculogenic ED.

\subsection{Safety}

No systemic complications in patients were seen. Three patients complained of brief penile pain shortly after injection, but there were no other local complications.

\subsection{Penile Color Doppler Sonography}

Six weeks after intracavernosal injection of Masport $₫$ IC, Doppler sonography of the penis was performed with $40 \mathrm{mg}$ papaverine injection. The mean PSV increase in the 100-unit group was 4.55 (from 26.45 to 31) and in the 50unit group was 0.46 (from 35.56 to 26.02). The results also showed that the increase in mean PSV due to the treatment was significant in the 100-unit group (P-value $<0.0001)$. Also, there was a significant difference (P-value $<0.0001$ ) between the two groups, so that patients treated with 100 units had a better response (Figure 1).

\subsection{Questionnaires and Response Rates}

4.4.1. IIEF Score Completed for Patients Six Weeks After Injection

Performance analysis was performed based on IIEF-EF improvement. At the first follow-up visit six weeks after Masport@ IC, the following definition was applied to responders: (i) Full responders: IIEF-EF-26; (ii) Relative responders: IIEF-EF $<26$ and increase in IIEF-EF against

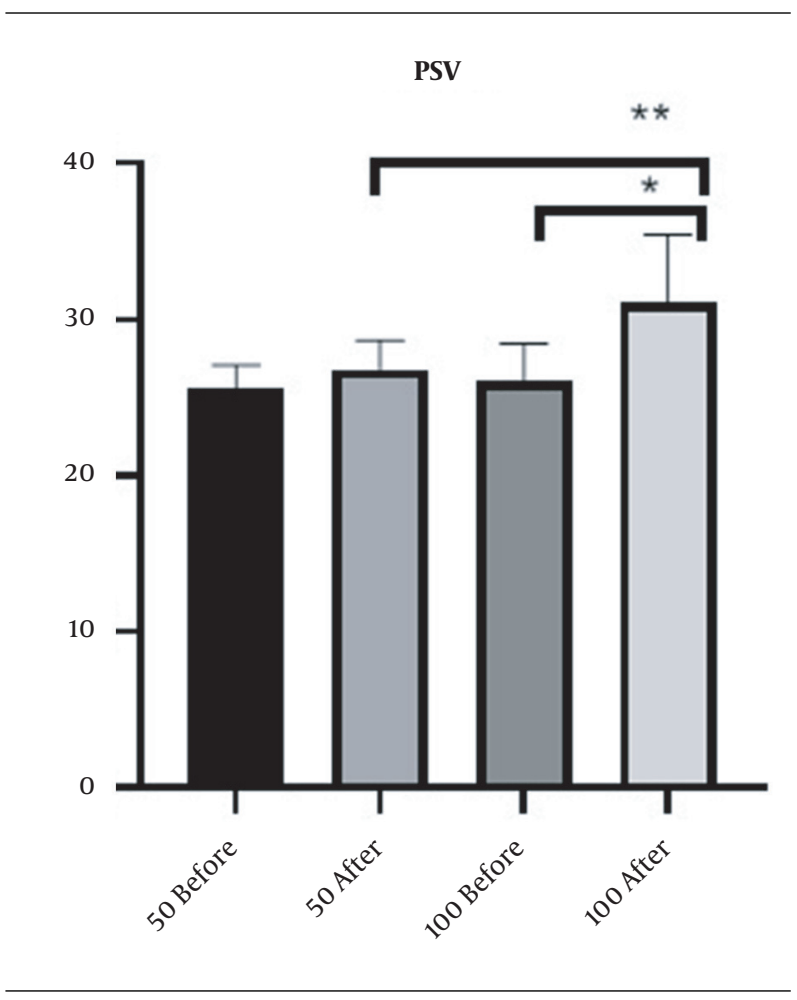

Figure 1. Comparison of PSV in two groups before and six weeks after injection

baseline- 4 and (III) Non-respondents: IIEF-EF $<26$ and increase in IIEF-EF against baseline $<4$ (11).

Six weeks after abobotulinumtoxinA IC, no patient was responding in the 50-unit group, but 18 (90\%) patients responded in the 100-unit group; however, no patient had a complete response and all responders were incomplete responders. Among the responders, 15 (75\%) were still using the same pharmacological ED treatment, and 5 (25\%) had modified the treatment prior to abobotulinumtoxinA IC. Three patients treated with sildenafil stopped taking it.

Within six weeks, the average increase in IEF-EF domain score was 4.3 (from 9.4 prior to Masport $®$ IC to 13.7) and 1 (from 8.1 prior to Masport@ IC to 9.1) in the 100-unit and 50unit groups, respectively. The increase in mean IIEF-EF due to treatment was significant between the two groups (Pvalue $<0.0001$ for 100 -unit group and P-value $<0.0369$ for 50-unit group); however, 100-unit group indicated a better response to treatment (Figure 2 ).

Six weeks after injection, SHIM score was completed for the patients. The mean SHIM score in 100-unit and 50unit groups increased by 4 (from 8.5 before Masport@ IC to 12.5 ) and 0.9 (from 8.1 before Masport $®$ IC to 9), respectively. The increase in mean SHIM score due to treatment in the 100-unit and 50-unit groups was significant (P-value < 0.0001 for the 100-unit group and P-value $<0.0191$ for the 


\section{IIEF}

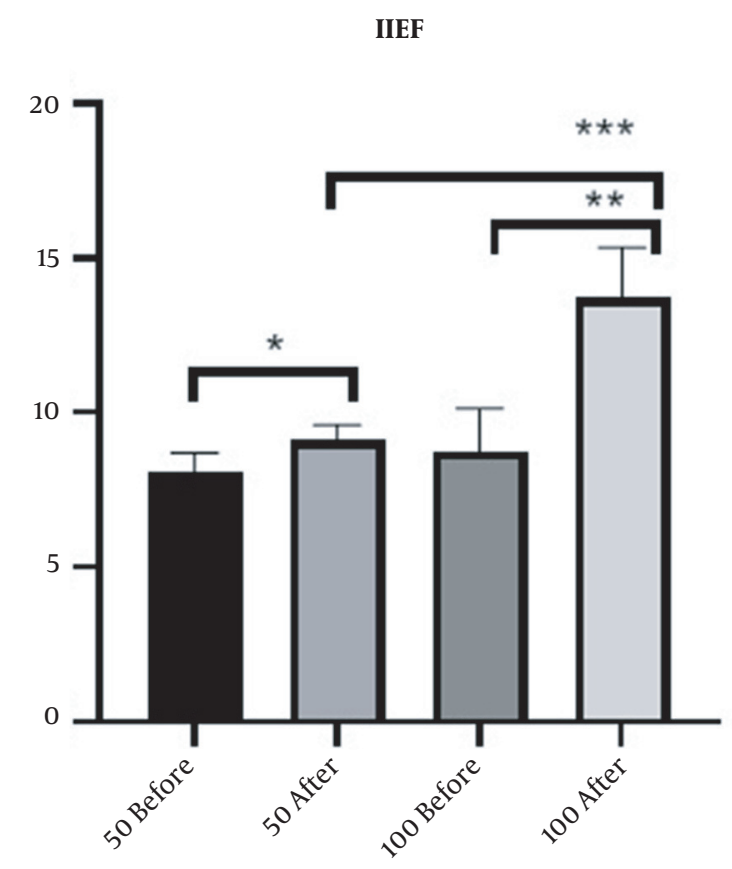

Figure 2. Comparison of IIEF in two groups before and six weeks after injection

50-unit group); however, 100-unit group indicated a better response to treatment (Figure 3)

EHS score was completed for patients six weeks after injection. The increase in mean EHS score in the 100-unit group was 0.5 (from 1.5 to 2 ) and in the 50-unit group was 0.2 (from 1.4 to 1.6 ). The results also showed that the increase in the mean EHS in 100-unit group due to treatment was significant (P-value $<0.0001)$ (Figure 4).

\section{Discussion}

At the National Institutes of Health (NIH) Conference on Impotence in 1992 it was stated that: "...because men, especially older men, are particularly sensitive to the social support of intimate relationships, withdrawal from these relationships due to ED may lead to having a significant negative impact on them (11).

Unfortunately, effective treatment for complex ED is still evolving despite the convenience of oral therapy. Some patients have contraindications to the use of a PDE5I, such as concomitant use of nitrate. Some patients cannot tolerate headache, visual and auditory changes. Also, $11-44 \%$ of patients do not respond to monotherapy with PDE5I. For this group of patients, the second line of treatment includes vacuum and ICI of vasoactive prostanoids. Other methods, including adrenoceptor antagonists, cen-

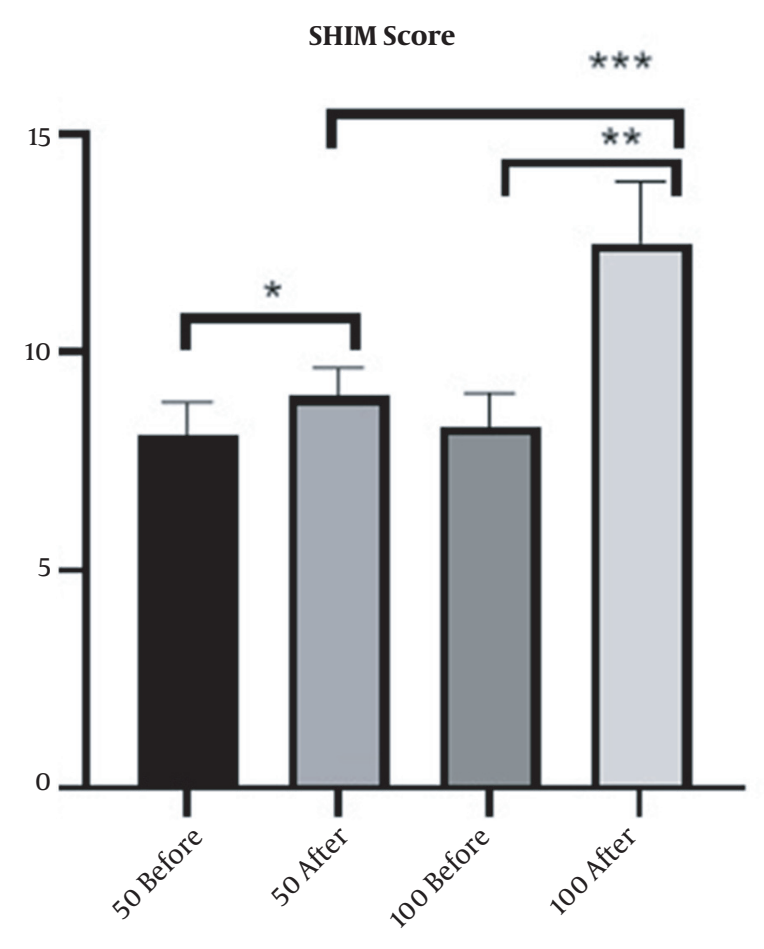

Figure 3. Comparison of SHIM score in two groups before and six weeks after injection

tral dopamine agonists, androgen replacement therapies, and other experimental drugs, have been used by physicians before surgery. However, satisfactory treatments for ED are still unknown and challenging (11). There are seven distinct subtypes of botulinum neurotoxins (BTX), including A, B, C, D, E, F, and G. The commercialized toxin types $A$ and $B$ have been approved by the Food and Drug Administration (FDA) (11). Various forms of botulinum toxin cause flaccid paralysis by inhibiting the secretion of acetylcholine in the presynaptic membrane. They bind to the receptor of a protein bound to the N-ethylmaleimidesensitive factor (SNARE) complex. SNARE complexes are an important group of polypeptides that bind to synaptic vesicles in the presynaptic membrane at the junction of muscle-nerves, thus allowing acetylcholine to be secreted into the synaptic space. Synaptosome-associated protein25 (SNAP-25) is a complex of SNARE that is affected by botulinum toxin (11).

Because the pathophysiology and the reason for patients' failure to respond to PDE5I is insufficient smooth muscle relaxation and venoocclusive disorder, any treatment that increases cavernosal smooth muscle relaxation can cause the patient to respond to PDE5I (11).

Since one of the main choices for the treatment of 


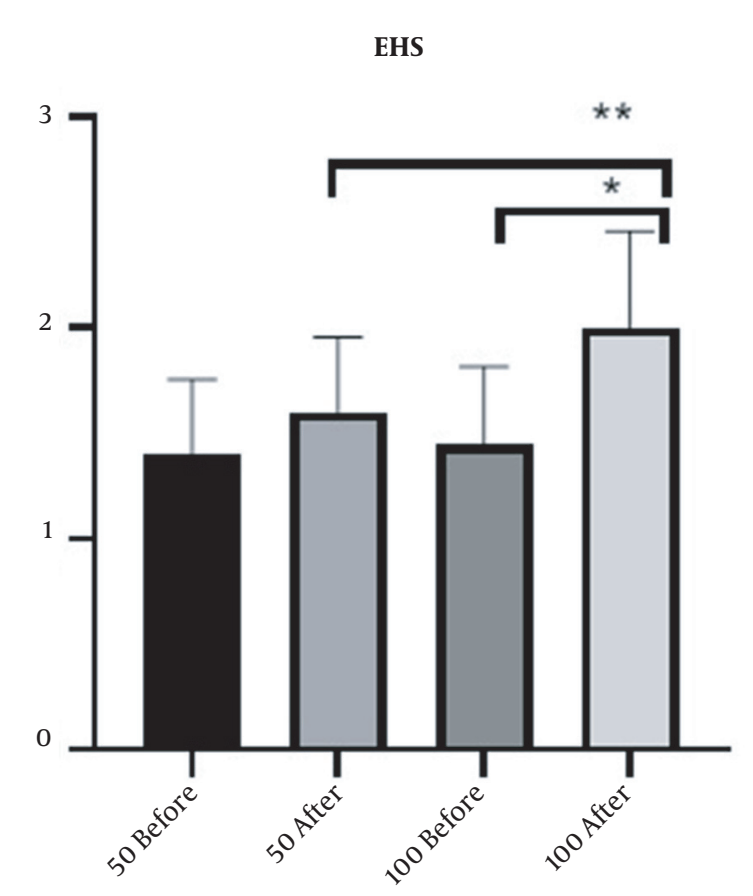

Figure 4. Comparison of EHS score in two groups before and six weeks after injection

these patients with vascular disorders resistant to PDE5I is surgery and invasive procedures, we used a safe dose of botulinum toxin for intracavernosal injection. We investigated this toxin in the treatment of patients with impotence resistant to the initial lines of treatment. In this study, according to IIEF criteria, all patients had severe vascular ED. We investigated this toxin in the treatment of patients with impotence resistant to the initial lines of treatment. In this study, according to IIEF criteria, all 40 patients had severe vascular ED. For all patients, IIEF, SHIM, and EHS questionnaires were completed. Then, the patients were randomly divided into two groups: ICI of a single dose of Masport $® 50$ units and single dose of 100 units.

Six weeks after intracavernosal injection of Masport, color Doppler sonography of the penis was performed with $40 \mathrm{mg}$ papaverine injection. There was an increase in mean PSV and EHS in the 100-unit group due to treatment (P-value $<0.0001)$, and there was a significant difference between the two groups (P-value $<0.0001$ ); but PSV and EHS did not change significantly in the 50-unit group before and after the treatment. Moreover, the mean IIEF-EF and SHIM score significantly increased due to treatment in the 100-unit and 50-unit groups (P-value $<0.0001$ for 100unit group), and there was a significant difference between the two groups (P-value $<0.0001$ ); also, there was a better response to treatment in the 100-unit group.

In a study conducted by Ghanem et al. in Egypt, 24 patients with severe vasculogenic ED resistant to PDEI5 or ICI (TRIMIX intracavernosal injection) were assessed. The results of penile Sono-Doppler two weeks after treatment were as follows: PSV increased from $24.6 \mathrm{CM} / \mathrm{S}$ to $34.9 \mathrm{CM} / \mathrm{S}$ ) $(\mathrm{P}=0.005)$; SHIM score improved six weeks after injection (from 5.58 to 10.25) ( $\mathrm{P}=0.0075)$; and the mean EHS improved from 2 to $2.75(\mathrm{P}=0.01)(11)$

According to the results of this study, a dose of 50 units of toxin was effective on ED resistant to PDEI5. According to the results, while 50-unit group improved SHIM score, 100-unit group improved all variables, including PSV, SHIM score, and EHS.

This was a retrospective uncontrolled case study with obvious limitations. Based on these preliminary findings, there is need for randomized placebo-controlled trials in order to further investigate the safety and efficacy of abobotulinumtoxinA IC to salvage PDE5Is and PGE1 IC insufficient responders when combined with existing pharmacological treatments. The response rate from the present pilot study showed that further multi-center randomized controlled trials with larger sample sizes should be conducted in the future.

\section{Conclusions}

The results of this study showed that intracavernosal injection of Botulinum Masport $₫$ toxin at a dose of 100 units in patients with vasculogenic ED resistant to PDE5I was slightly effective in improving sexual function and ultrasound indices. Further studies are needed to confirm our results.

\section{Footnotes}

Authors' Contribution: Study concept and design: N.B. and S.M.; Analysis and interpretation of data: M.D. and D.K.; Drafting of the manuscript: S.M.; Critical revision of the manuscript for important intellectual content: N.B., D.K., and M.D.; Statistical analysis: N.B.

\section{Clinical Trial Registration Code: U-99269.}

Conflict of Interests: The authors declared no conflict of interest.

Ethical Approval: This study was approved by the Ethical Committee of Ahvaz Jundishapur University of Medical Sciences, Iran (code: IR.AJUMS.REC.1399.105).

Funding/Support: This study was done under the support of the Research and development deputy, Ahvaz Jundishapur University of Medical Sciences, Ahvaz, Iran 
(grant No \#U-99269). We thank the Imam Khomeini Clinical Research and Development unit for the scientific support of the research.

Informed Consent: Informed consent was obtained from all patients.

\section{References}

1. McCabe MP, Sharlip ID, Lewis R, Atalla E, Balon R, Fisher AD, et al. Incidence and prevalence of sexual dysfunction in women and men: a consensus statement from the Fourth International Consultation on Sexual Medicine 2015.J Sex Med. 2016;13(2):144-52.

2. Feldman HA, Goldstein I, Hatzichristou DG, Krane RJ, McKinlay JB. Impotence and its medical and psychosocial correlates: results of the Massachusetts Male Aging Study.J Jrol.1994;151(1):54-61.

3. Araujo AB, Esche GR, Kupelian V, O'Donnell AB, Travison TG, Williams $\mathrm{RE}$, et al. Prevalence of symptomatic androgen deficiency in men. J Clin Endocrinol Metab. 2007;92(11):4241-7. doi: 10.1210/jc.2007-1245. [PubMed: 17698901].

4. Gratzke C, Angulo J, Chitaley K, Dai YT, Kim NN, Paick JS, et al. Anatomy, physiology, and pathophysiology of erectile dysfunction. J Sex Med. 2010;7(1 Pt 2):445-75. doi: 10.1111/j.1743-6109.2009.01624.x. [PubMed: 20092448].
5. Burnett AL, Nehra A, Breau RH, Culkin DJ, Faraday MM, Hakim LS, et al. Erectile Dysfunction: AUA Guideline.JUrol.2018;200(3):633-41. doi: 10.1016/j.juro.2018.05.004. [PubMed: 29746858].

6. Hatzimouratidis K, Giuliano F, Moncada I, Muneer A, Salonia A, Verze P. EAU guidelines on male sexual dysfunction. Arnhem: EAU Guidelines Office. 2018.

7. Sommer F, Engelmann U. Future options for combination therapy in the management of erectile dysfunction in older men. Drugs Aging. 2004;21(9):555-64. doi: 10.2165/00002512-200421090-00001. [PubMed: 15260511].

8. M. Yaiesh S, F. Almutairi M, E. Al-Terki A, F. Al-Shaiji T. Urological Applications of Botulinum Toxin A. Botulinum Toxin. IntechOpen; 2018. doi: 10.5772/intechopen.79555.

9. Ghanem H, Raheem AA, AbdelRahman IFS, Johnson M, AbdelRaheem T. Botulinum Neurotoxin and Its Potential Role in the Treatment of Erectile Dysfunction. Sex Med Rev. 2018;6(1):135-42. doi: 10.1016/j.sxmr.2017.07.008. [PubMed: 28843941].

10. De Young L, Campbell J, Radomski S, Alzubaidi R, Brock G. 142 Intracavernosal Injection of Botulinum Toxin to Improve Erectile Function in Older Rats.J Sex Med. 2017;14(1):S40-1. doi: 10.1016/j.jsxm.2016.11.091.

11. Yang M, Ni X, Sontag A, Litman HJ, Rosen RC. Nonresponders, partial responders, and complete responders to PDE5 inhibitors therapy according to IIEF criteria: validation of an anchor-based treatment responder classification. J Sex Med. 2013;10(12):3029-37. doi: 10.1111/jsm.12335. [PubMed: 24125113]. 